\title{
Nonadiabatic level crossing in resonant and nonresonant neutrino oscillations
}

\author{
M. Kachelrieß \\ TH Division, CERN, 1211 Geneva 23, Switzerland
}

R. Tomàs

Instituto de Fisica Corpuscular - C.S.I.C. - Universitat de València, 46071 València, Spain

(Received 9 April 2001; published 27 August 2001)

\begin{abstract}
We study neutrino oscillations and the level-crossing probability $P_{\mathrm{LSZ}}=\exp \left(-\gamma_{n} \mathcal{F}_{n} \pi / 2\right.$ ) (LSZ stands for Landau-Stückelberg-Zener) in power-law-like potential profiles $A(r) \propto r^{n}$. After showing that the resonance point coincides only for a linear profile with the point of maximal violation of adiabaticity, we point out that the "adiabaticity" parameter $\gamma_{n}$ can be calculated at an arbitrary point if the correction function $\mathcal{F}_{n}$ is rescaled appropriately. We present a new representation for the level-crossing probability, $P_{\mathrm{LSZ}}=\exp \left(-\kappa_{n} \mathcal{G}_{n}\right)$, which allows a simple numerical evaluation of $P_{\text {LSZ }}$ in both the resonant and nonresonant cases, and where $\mathcal{G}_{n}$ contains the full dependence of $P_{\mathrm{LSZ}}$ on the mixing angle $\vartheta$. As an application we consider the case $n=-3$ important for oscillations of supernova neutrinos.
\end{abstract}

DOI: 10.1103/PhysRevD.64.073002 PACS number(s): 14.60.Lm, 14.60.Pq, 26.65.+t, 97.60.Bw

\section{INTRODUCTION}

The analytical study of nonadiabatic neutrino oscillations has a long history. Soon after the discovery of "resonant" neutrino flavor conversions by Mikheev and Smirnov [1], the leading nonadiabatic effects were calculated for a linear potential profile in Ref. [2]. They were obtained in the form of the Landau-Stückelberg-Zener (LSZ) crossing probability [3]

$$
P_{\mathrm{LSZ}}=\exp \left(-\frac{\pi \gamma}{2}\right)
$$

The adiabaticity parameter $\gamma$ for a linear profile is

$$
\gamma=\frac{\Delta S^{2}}{2 E C|\mathrm{~d} \ln A / \mathrm{d} r|_{0}},
$$

where $E$ is the energy, $\Delta=m_{2}^{2}-m_{1}^{2}>0, \quad S=\sin (2 \vartheta), C$ $=\cos (2 \vartheta), \vartheta \in[0: \pi / 2]$ is the (vacuum) mixing angle, and $A=2 E V=2 \sqrt{2} G_{F} N_{e} E$ is the induced mass squared term for the electron neutrino. The parameter $\gamma$ has to be evaluated at the so-called resonance point $r_{0}$, i.e. the point where the mixing angle in matter is $\vartheta_{m}=\pi / 4$. For a linear profile, adiabaticity is maximally violated for $\vartheta_{m}=\pi / 4$ and, therefore, the probability that a neutrino jumps from one branch of the dispersion relation to the other is indeed maximal at $r_{0}$.

Later, Kuo and Pantaleone [4] derived the LSZ crossing probability for an arbitrary power-law-like profile, $A \propto r^{n}$. They also found that in this case the dependence on the neutrino masses and energies can be factored out, while the effect of a nonlinear profile can be encoded into a correction function $\mathcal{F}_{n}$,

$$
P_{\mathrm{LSZ}}=\exp \left(-\frac{\pi \gamma_{n}}{2} \mathcal{F}_{n}(\vartheta)\right)
$$

which depends only on $\vartheta$ and $n$. The "adiabaticity" parameter $\gamma_{n}$ still has to be evaluated at $r_{0}$ although, as we will show, it does not coincide with the point of maximal viola- tion of adiabaticity (PMVA) for $n \neq 1$. An unsatisfactory feature of Eq. (3) is its restricted range of applicability: the resonance condition $\vartheta_{m}=\pi / 4$ has a solution only if $\vartheta$ $<\pi / 4$ for neutrinos or if $\vartheta>\pi / 4$ for antineutrinos, respectively. Therefore, it is not possible to calculate analytically, e.g., the survival probability of supernova antineutrinos in the quasiadiabatic regime assuming a normal mass hierarchy.

The purpose of this paper is twofold. First, we discuss the physical significance of the resonance point $r_{0}$. We show that the product $\gamma_{n} \mathcal{F}_{n}$ can be evaluated at an arbitrary point and conclude that the "resonance" point $r_{0}$ has, for a general profile $n \neq 1$, no particular physical meaning: neither does it describe the point of maximal violation of adiabaticity nor is it necessary to calculate the "adiabaticity" parameter $\gamma_{n}$ at $r_{0}$. Second, we propose a new representation for $P_{\mathrm{LSZ}}$ that is valid for all $\vartheta$ and allows an easy numerical evaluation. As an application, we consider the case $n=-3$ which is important for oscillations of supernova neutrinos.

\section{RESONANCE POINT VS MAXIMAL VIOLATION OF ADIABATICITY}

As a starting point for our discussion we use the evolution equation for the medium states $\widetilde{\psi}$ first given in Ref. [5]:

$$
\frac{d}{d r}\left(\begin{array}{c}
\widetilde{\psi}_{1} \\
\widetilde{\psi}_{2}
\end{array}\right)=\left(\begin{array}{cc}
i \Delta_{m} /(4 E) & -\vartheta_{m}^{\prime} \\
\vartheta_{m}^{\prime} & -i \Delta_{m} /(4 E)
\end{array}\right)\left(\begin{array}{c}
\widetilde{\psi}_{1} \\
\widetilde{\psi}_{2}
\end{array}\right) .
$$

Here

$$
\Delta_{m}=\sqrt{(A-\Delta C)^{2}+(\Delta S)^{2}}
$$

denotes the difference between the effective mass of the two neutrino states in matter, $\vartheta_{m}$ is the mixing angle in matter with

$$
\tan 2 \vartheta_{m}=\frac{\Delta S}{\Delta C-A}
$$




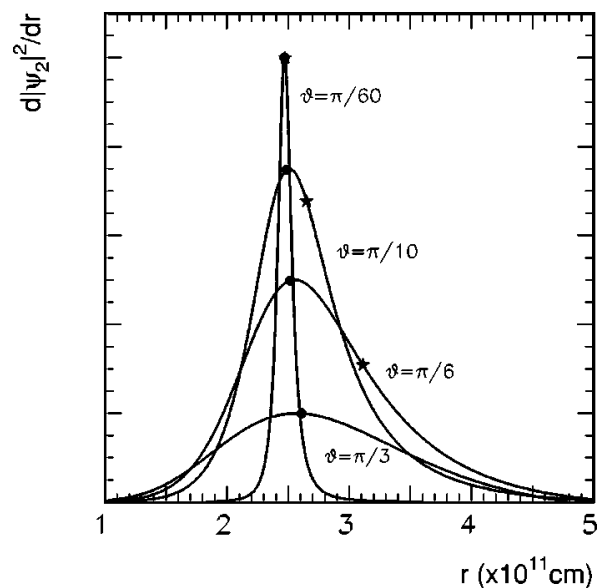

FIG. 1. Change of the survival probability $d p(r) / d r$ of a neutrino produced at $r=0$ as $\nu_{2}$ together with the point of maximal violation of adiabaticity (dots) and the resonance point (stars) for a power-law profile $A \propto r^{-3}$. The height of the different curves is rescaled.

and $\vartheta_{m}^{\prime}=d \vartheta_{m} / d r$.

The evolution of the neutrino state is adiabatic at a given $r$, if the diagonal terms are large compared to the offdiagonal ones, $\left|\Delta_{m}\right| \gg\left|4 E \vartheta_{m}^{\prime}\right|$. Thus the point where adiabaticity is maximally violated is given by the minimum of $\Delta_{m} / \vartheta_{m}^{\prime}$. Differentiating [6]

$$
\frac{\Delta_{m}}{\vartheta_{m}^{\prime}}=\frac{2 \Delta^{2} S^{2}}{\sin ^{3} 2 \vartheta_{m}} \frac{1}{d A / d r}
$$

for the case of a power-law profile, $A(r) \propto r^{n}$, we find the minimum at

$$
\begin{aligned}
& \cot \left(2 \vartheta_{m}-2 \vartheta\right)+2 \cot \left(2 \vartheta_{m}\right)-\frac{1}{n}\left[\cot \left(2 \vartheta_{m}-2 \vartheta\right)\right. \\
& \left.-\cot \left(2 \vartheta_{m}\right)\right]=0 .
\end{aligned}
$$

For $n=1$, the PMVA is at $\vartheta_{m}=\pi / 4$ for all $\vartheta$. Thus, in the region where the resonance point is welldefined, the PMVA and $r_{0}$ coincide. However, in the general case, $n \neq 1$, the PMVA agrees with the resonance point only for $\vartheta=0$. Finally, we recover the result of Ref. [6] for an exponential profile in the limit $n \rightarrow \pm \infty$.

In Fig. 1, we show the change of the survival probability $d p(r) / d r=d\left|\tilde{\psi}_{2}(r)\right|^{2} / d r$ of a neutrino produced at $r=0$ as $\nu_{2}$ as a function of $r$, together with the point of maximal violation of adiabaticity predicted by Eq. (8) and the resonance point for a power-law profile $A \propto r^{-3}$. It can clearly be seen that Eq. (8) accurately describes the most probable position of the level crossing, while the resonance point predicts a transition in less and less dense regions, until for $\vartheta$ $=\pi / 4$ the concept of a resonant transition breaks down completely. If the true profile is only approximately a power law, its exponent should therefore be determined by the region around the PMVA, not by the region around the resonance point.

\section{CORRECTION FUNCTIONS $\mathcal{F}_{n}$}

We now briefly recall the calculation of the leading term to the crossing probability within the WKB formalism [4]. In the ultrarelativistic limit and omitting an overall phase, the WKB formula results in

$$
\ln P_{\mathrm{LSZ}}=-\frac{1}{E} \mathfrak{I} \int_{x_{1}\left(A_{1}\right)}^{x_{2}\left(A_{2}\right)} d x \Delta_{m}
$$

where $A_{2}=\Delta e^{2 i \vartheta}$ is the branch point of $\Delta_{m}$ in the upper complex $x$ plane. We identify the physical coordinate $r$ $\in[0: \infty]$ with the positive part of the real $x$ axis, i.e., we consider a neutrino state produced at small but positive $x$ propagating to $x=\infty$. Then a convenient choice for $A_{1}$ is to use the real part of $A_{2}$ for $C>0$, i.e., the "resonance" point $A_{1}=\Delta C$. However, we stress that this choice has technical reasons, and makes sense only for $C>0$ : consider for instance the simplest case $n=1$. Then both the integration path chosen and the branch cut are for $C<0$ in the half-plane $\mathfrak{R}(x)<0$. The physical interpretation is therefore that an antineutrino state created at small but negative $x$ propagates to $-\infty$. However, this case is equivalent to a neutrino state propagating with $C>0$ in the right half-plane and therefore contains no new information. Thus we expect the correction functions $\mathcal{F}_{n}$ obtained with the integration path from $\Delta C$ to $\Delta \mathrm{e}^{2 \mathrm{i} \vartheta}$ to be functions with period $\pi / 4$ and to be valid only in the resonant region.

After the substitution $A=\Delta(B+C)$,

$$
\ln P_{\mathrm{LSZ}}=-\frac{\Delta}{E} \mathfrak{I} \int_{0}^{\mathrm{i} S} d B \frac{d x}{d B}\left(B^{2}+S^{2}\right)^{1 / 2},
$$

one has to expand the Jacobian $d x / d B$ in a power series in order to solve the $B$ integrals. Kuo and Pantaleone chose as expansion point the "resonance" point $B_{0}=0$, because it leads to the simplest result. It is this choice, arbitrary from a physical point of view, that leads to the evaluation of the parameter $\gamma_{n}$ at $B_{0}=0$. In general, a change of $B_{0}$ in the definition of $\gamma_{n}$ will be compensated for by such a change in the correction function $\mathcal{F}_{n}$ that the physical observable $P_{\text {LSZ }}$ is independent of $B_{0}$ [7].

The final result for the correction functions given by Kuo and Pantaleone was

$$
\mathcal{F}_{n}(\vartheta, 0)=2 \sum_{m=0}^{\infty}\left(\begin{array}{c}
1 / n-1 \\
2 m
\end{array}\right)\left(\begin{array}{c}
1 / 2 \\
m+1
\end{array}\right)[\tan (2 \vartheta)]^{2 m}
$$

This series for $\mathcal{F}_{n}(\vartheta, 0)$ converges only for $\vartheta<\pi / 8$ and is therefore not suited, even in the resonant region, for a numerical evaluation in the phenomenologically most interesting case of maximal or nearly maximal mixing. Representing the series as a hypergeometric function,

$$
\mathcal{F}_{n}(\vartheta, 0)={ }_{2} F_{1}\left(\frac{n-1}{2 n}, \frac{2 n-1}{2 n} ; 2 ;-\tan ^{2}(2 \vartheta)\right),
$$

however, one can use the Euler integral representation [8] of ${ }_{2} F_{1}$ as the analytical continuation for all $\vartheta \in[0: \pi / 4]$. 
We now discuss the nonresonant case, $\vartheta \in[\pi / 4: \pi / 2]$. The expression $\gamma_{n}(0) \mathcal{F}_{n}(0, \vartheta)$ becomes ill defined in this region for several reasons: the assumption $C \geqslant 0$ used in the derivation for $\mathcal{F}_{n}(\vartheta, 0)$ is not fulfilled, and the "resonance" condition $B=0$ or $A=\Delta C$ has no solution for $\vartheta$ $\in[\pi / 4: \pi / 2]$. Moreover, the integration path used in Eq. (10) can be for $C<0$ in the unphysical region $x<0$. However, we know from the results above that the crossing probability is also nonzero in the nonresonant case. Also, the WKB method should work independently of the sign of $C$ as long as the evolution of the neutrino state is not strongly nonadiabatic.

We now show that it is not necessary to evaluate $\gamma_{n} \mathcal{F}_{n}$ at the resonance point $B_{0}=0$. Requiring the invariance of $P_{\mathrm{LSZ}}$ against variations of $B_{0}$,

$$
\mathcal{F}_{n} \frac{\partial \gamma_{n}}{\partial B_{0}}+\gamma_{n} \frac{\partial \mathcal{F}_{n}}{\partial B_{0}}=0
$$

with

$$
\frac{\partial \gamma_{n}}{\partial B_{0}}=\frac{\gamma}{n\left(B_{0}+C\right)}
$$

we obtain a differential equation for $\mathrm{d} \mathcal{F} / \mathrm{d} B_{0}$. Its solution

$$
\mathcal{F}_{n}\left(\vartheta, B_{0}\right)=\left(\frac{C}{B_{0}+C}\right)^{1 / n} \mathcal{F}_{n}(\vartheta, 0)
$$

allows us to rescale the correction functions obtained for $B_{0}=0$ to arbitrary $B_{0}$. Therefore, we can now also calculate $P_{\mathrm{LSZ}}$ for the nonresonant region in the two cases in which the function $\mathcal{F}_{n}$ is known for all $\vartheta$.

For a $1 / r$ profile, $A(r)=2 E V_{0}\left(R_{0} / r\right)$, the correction function is [4]

$$
\mathcal{F}_{-1}(0, \vartheta)=\frac{\left[1-\tan ^{2}(\vartheta)\right]^{2}}{1+\tan ^{2}(\vartheta)} .
$$

If we want to calculate the level-crossing probability in both resonant and nonresonant cases, we have to choose $B_{0} \geqslant 1$, e.g.

$$
\mathcal{F}_{-1}(1, \vartheta)=\frac{1+C}{C} \mathcal{F}_{-1}(0, \vartheta)
$$

the crossing probability follows as

$$
P_{\mathrm{LSZ}}=\exp \left\{-2 \pi R_{0} V_{0} \sin ^{2}(\vartheta)\right\} .
$$

As an example, in Fig. 2 we compare the results of a numerical solution of the Schrödinger equation (4) with the analytical calculation of $P_{\text {LSZ }}$ using the rescaled $\mathcal{F}_{-1}$ function [9]. The agreement between the different methods is excellent.

In the limit $n \rightarrow \pm \infty$, which corresponds to an exponential potential profile, the scale factor $\left[C /\left(B_{0}+C\right)\right]^{1 / n}$ goes to 1 and the correction function becomes independent of $B_{0}$, as it should. The resulting crossing probability is

$$
P_{\mathrm{LSZ}}=\exp \left\{-\frac{\pi \Delta R_{0}}{E} \sin ^{2}(\vartheta)\right\} \text {. }
$$

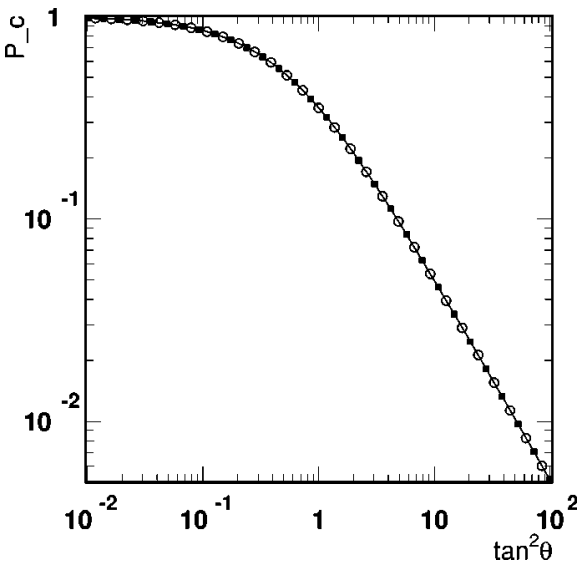

FIG. 2. Crossing probability $P_{c}$ for $A \propto 1 / r$ with $R_{0} V_{0}=0.2$ : numerically (solid line), with $\mathcal{F}_{-1}$ evaluated at $B_{0}=1$ (squares) and $\mathcal{G}_{-1}$ (circles).

In Refs. [10], this expression was derived by solving Schrödinger's equation directly. In these works, it was assumed that the obtained expression is valid only in the resonant region, and only recently was it pointed out that it is valid for all $\vartheta$ [11]. Note also that $\ln P_{\mathrm{LSZ}}$ has the same, very simple dependence on $\vartheta$, for both $n=-1$ and $n \rightarrow \pm \infty$.

\section{CORRECTION FUNCTIONS $\mathcal{G}_{\boldsymbol{n}}$}

We start directly from Eq. (9), but now as an integration path in the complex $x$ plane we use the part of the circle centered at zero starting at $A_{1}=\Delta$ and going to the end of the branch cut at $A_{2}=\Delta e^{2 i \vartheta}$. Substituting $x=R_{0}\left(\Delta / A_{0}\right)^{1 / n} e^{i \varphi}$ in the case of a potential $A=A_{0}\left(r / R_{0}\right)^{n}$, we can factor out the $\vartheta$ dependence of $P_{\mathrm{LSZ}}$ into functions $\mathcal{G}_{n}$,

$$
\ln P_{\mathrm{LSZ}}=-\kappa_{n} \mathcal{G}_{n}(\vartheta),
$$

where

$$
\kappa_{n}=\left(\frac{\Delta}{E}\right)\left(\frac{\Delta}{A_{0}}\right)^{1 / n} R_{0}
$$

is independent of $\vartheta$, and

$$
\mathcal{G}_{n}(\vartheta)=\left|\Re \int_{0}^{2 \vartheta / n} d \varphi e^{i \varphi}\left[\left(e^{i n \varphi}-C\right)^{2}+S^{2}\right]^{1 / 2}\right| .
$$

The functions $\mathcal{G}_{n}$ are well suited for numerical evaluation, and always correspond to a neutrino state propagating in the physical part of the $x$ plane, $x>0$. Therefore, they have, in contrast to the $\mathcal{F}_{n}$ functions, a period $\pi / 2$, and are valid for all $\vartheta$. In Fig. 2, the results of this new representation are compared with those obtained above for the case $n=-1$.

\section{OSCILLATIONS OF SUPERNOVA NEUTRINOS}

The potential profile $A(r)$ in supernova $(\mathrm{SN})$ envelopes can be approximated by a power law with $n \approx-3$, and $V(r)=1.5 \times 10^{-9} \mathrm{eV}\left(10^{9} \mathrm{~cm} / r\right)^{-3}$ [12]. Since only $\bar{\nu}_{e}$ were detected from SN 1987A, and since also in the case of 


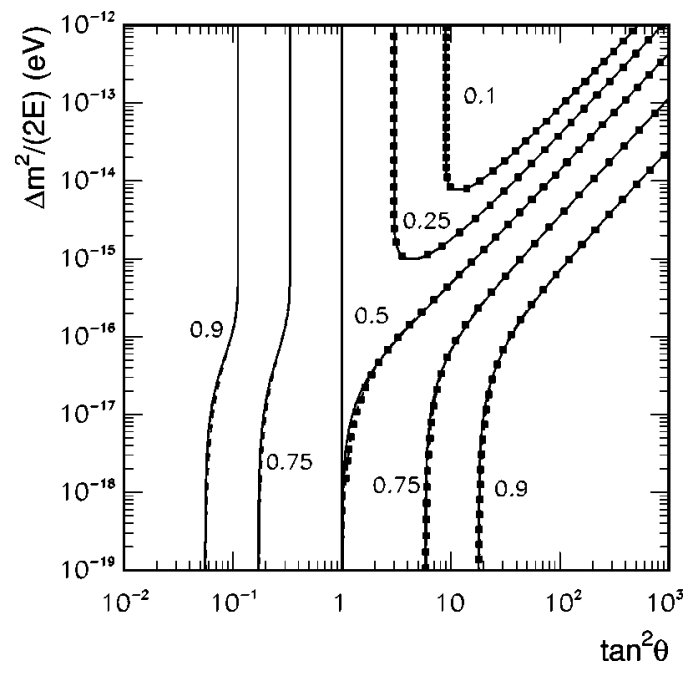

FIG. 3. Contours of constant survival probability $P_{e e}^{--}$, numerically (solid lines), with $\mathcal{G}_{-3}$ (dashed lines) and $\mathcal{F}_{-3}$ (squares, only for $\vartheta>\pi / 4$ ), for $A \propto r^{-3}$ as given in the text.

a future galactic SN the $\bar{\nu}_{e}$ flux will dominate the observed neutrino signal, an analytical expression for $P_{c}$ valid in the nonresonant part of the mixing space is especially useful [7]. The probability for $\bar{\nu}_{e}$ to arrive at the surface of the Earth can be written as an incoherent sum of probabilities [13]:

$$
P_{e e}^{--}=\left(1-P_{c}\right) \cos ^{2} \vartheta+P_{c} \sin ^{2} \vartheta
$$

In Fig. 3, we compare the results of a numerical solution of the Schrödinger equation (4) with an analytical calculation of $P_{e e}^{--}$using the $\mathcal{G}_{-3}$ and $\mathcal{F}_{-3}$ functions. The latter is shown only for its range of applicability, $\vartheta>\pi / 4$. The agreement between the two methods using the WKB approach is again excellent (for $\vartheta>\pi / 4$ ). Generally, these two methods also agree very well with the results of the numerical solution of the Schrödinger equation; there are only small deviations in the regions where the contours change their slope.

\section{SUMMARY}

We have discussed nonadiabatic neutrino oscillations in general power-law potentials $A \propto r^{n}$. We have found that the conventional splitting of $\ln P_{\text {LSZ }}$ in an "adiabaticity" parameter $\gamma_{n}$, evaluated at the resonance point and a correction function $\mathcal{F}_{n}$, is misleading for $n \neq 1$ : the level-crossing probability does not have a maximum at $r_{0}$, nor does this splitting allow a calculation of $P_{\mathrm{LSZ}}$ in the nonresonant region. We have proposed a new representation for $P_{\mathrm{LSZ}}$ that avoids these problems and is hopefully useful for the investigations of oscillations of supernova neutrinos.

\section{ACKNOWLEDGMENTS}

We are grateful to Uli Nierste for helpful discussions. R.T. would like to thank the Theory Division at CERN for hospitality and the Generalitat Valenciana for a grant. This work was also supported by Spanish DGICYT Grant No. PB980693, by the European Commission TMR networks ERBFMRXCT960090 and HPRN-CT-2000-00148, and by the European Science Foundation network N. 86.
[1] S.P. Mikheev and A.Yu. Smirnov, Yad. Fiz. 42, 1441 (1985) [Sov. J. Nucl. Phys. 42, 913 (1985)]; Nuovo Cimento Soc. Ital. Fis., C 9, 17 (1986).

[2] W.C. Haxton, Phys. Rev. Lett. 57, 1271 (1986); S.J. Parke, ibid. 57, 1275 (1986).

[3] L. Landau, Phys. Z. Sowjetunion 2, 46 (1932); E.C.G. Stückelberg, Helv. Phys. Acta 5, 369 (1932); C. Zener, Proc. R. Soc. London, Ser. A 137, 696 (1932).

[4] T.K. Kuo and J. Pantaleone, Phys. Rev. D 39, 1930 (1989).

[5] S.P. Mikheev and A.Yu. Smirnov, Zh. Éksp. Teor. Fiz. 92, 404 (1987) [Sov. Phys. JETP 65, 230 (1987)].

[6] A. Friedland, Phys. Rev. D 64, 013008 (2001).

[7] For a detailed discussion, see M. Kachelrieß, R. Tomàs, and J. W. F. Valle (unpublished).

[8] See, e.g., relation (15.3.1) of Handbook of Mathematical Functions, edited by M. Abramowitz and I. A. Stegun (National Bureau of Standards, Washington DC, 1964).
[9] We recall that the Landau-Stückelberg-Zener formula is only valid for small deviations from adiabaticity. Generally, the WKB formula [Eq. (3)] should be replaced [4] by

$$
P_{c}=\frac{\exp \left(-\frac{\pi \gamma}{2} \mathcal{F}\right)-\exp \left(-\frac{\pi \gamma}{2} \mathcal{F}^{\prime}\right)}{1-\exp \left(-\frac{\pi \gamma}{2} \mathcal{F}^{\prime}\right)},
$$

where $\mathcal{F}^{\prime}=\mathcal{F} / \sin ^{2} \vartheta$.

[10] S.T. Petcov, Phys. Lett. B 200, 373 (1988); P.I. Krastev and S.T. Petcov, ibid. 207, 64 (1988); 214, 661(E) (1988); S.T. Petcov, ibid. 214, 139 (1988).

[11] A. de Gouvêa, A. Friedland, and H. Murayama, J. High Energy Phys. 03, 009 (2001).

[12] K. Nomoto and M. Hashimoto, Phys. Rep. 163, 13 (1988).

[13] Note that the expressions for antineutrinos can be obtained from those for neutrinos by changing $\vartheta \rightarrow \pi / 2-\vartheta$ in $P_{c}$. 